Research Paper

\title{
Microvascular Damage Is Involved in the Pathogenesis of Heroin Induced Spongiform Leukoencephalopathy
}

\author{
Ruixue Yin ${ }^{1}$, Changjun $\mathrm{Lu}^{2}$, Qiang Chen ${ }^{3}$, Jianzhong Fan¹, Jiangyang $\mathrm{Lu}^{4}{ }^{\bowtie}$ \\ 1. Department of Rehabilitation Medicine, Nanfang Hospital, Southern Medical University, Guangzhou 510515, China; \\ 2. Department of Neurology, Liuzhou Traditional Chinese Medicine Hospital, Liuzhou 545001, China; \\ 3. Department of Neurology, Ningbo Beilun people Hospital, Ningbo 315806, China; \\ 4. Department of Pathology, First Affiliated Hospital of General Hospital of PLA, Beijing 100048, China.
}

$\bowtie$ Corresponding author: Jiangyang Lu, Tel: 010-66867432; lujy@263.net; shenzt@smu.edu.cn. Department of Pathology, First Affiliated Hospital of General Hospital of PLA, Beijing 100048, China.

() Ivyspring International Publisher. This is an open-access article distributed under the terms of the Creative Commons License (http://creativecommons.org/ licenses/by-nc-nd/3.0/). Reproduction is permitted for personal, noncommercial use, provided that the article is in whole, unmodified, and properly cited.

Received: 2012.07.06; Accepted: 2013.01.16; Published: 2013.02.0I

\begin{abstract}
Objective: To investigate whether microvascular damage is involved in the pathogenesis of heroin induced spongiform leukoencephalopathy (HSLE).

Methods: The brain tissues were collected from 4 HSLE patients and 5 controls and then fixed in $4 \%$ paraformaldehyde. The frontal lobe, corpus callosum and cerebellum were separated. The expressions of myelin base protein (MBP) and CD34 were detected by immunohistochemistry. TUNEL staining was applied to detect cell apoptosis. The correlation between microvascular changes and pathological vacuoles was evaluated.

Results: No obvious abnormalities were found in the brain of controls. Immunohistochemistry for MBP showed the collapse and fracture of myelin sheath and vacuole formation in the subcortical white matter, corpus callosum, and cerebellar white matter of HSLE patients. TUNEL staining showed the number of apoptotic cells in the cerebellar white matter and corpus callosum of HSLE patients was significantly higher than that in controls $(F=389.45 \mathrm{I}, P<0.00 \mathrm{I})$. Masson's trichrome staining revealed vacuolar degeneration in the cerebral white matter of HSLE patients, and the vacuoles were distributed around the microvessels. Immunohistochemistry revealed CD34 positive cells were seldom found besides the vessels in the cerebellar white matter and corpus callosum of HSLE patients, but a variety of CD34 positive cells was found in the vascular wall of controls $(F=838.500, P<0.001)$.

Conclusion: Apoptosis of oligodendrocytes may be related to the HSLE. Cerebral vascular injury and microcirculation dysfunction are involved in the pathogenesis of HSLE. The interrelation between apoptosis of oligodendrocytes and the microvascular damage are required to be studied in future investigations.
\end{abstract}

Key words: heroin induced spongiform leukoencephalopathy; microvessel; apoptosis; demyelination; myelin base protein.

\section{Introduction}

Previous imaging has shown that heroin induced spongiform leukoencephalopathy (HSLE) is pathologically characterized by extensive and symmetrical lesions in the white matter, which is mainly found in the cerebellum, posterior limb of internal capsule, splenium of the corpus callosum, parietal lobe and occipital lobe [1]. The increase in myelin base protein (MBP) has been found in the cerebral spinal fluid 
(CSF) of HSLE patients [2]. The pathogenesis of HSLE was found to be related to the demyelination of the central nervous system (CNS). Single photon emission computed tomography (SPECT) for detection of cerebral perfusion indicates the reduction in blood flow in the cerebral white matter of HSLE patients accompanied by involvement of cerebral grey matter to different extents [3]. There is evidence showing that ischemia/hypoxia, chemical poisoning and radiographic exposure may induce the secondary apoptosis of oligodendrocytes, finally resulting in axon demyelination and loss of transduction $[4,5]$. The damage to the cerebral white matter in HSLE patients is similar to that in hypoxic - ischemic encephalopathy patients, while HSLE is different from delayed post-anoxic leukoencephalopathy. The most involved parts of the post-anoxic leukoencephalopathy are cerebral cortex, hippocampus, cerebellum, thalamus, caudate nucleus and brainstem motor nuclei, while HSLE mainly involves the white matter.

Our previous study showed that the vacuolar degeneration in the white matter of HSLE patients was related to the oligodendrocyte apoptosis induced demyelination. Whether there is alteration in the cerebral microvessels of HSLE patients and whether this alteration is related to the oligodendrocyte apoptosis are still unclear. In the present study, immunohistochemistry for MBP was performed to detect the demyelination, TUNEL staining to measure the apoptosis, and Masson's trichrome staining to detect the cerebral microvessels. In addition, the correlation between the alteration of microvessels and pathological vacuolar degeneration was evaluated. Our findings may shed light on the pathogenesis of HSLE.

\section{Materials and methods}

\section{Patients}

The brain was collected from 4 HSLE patients in Nanfang Hospital from July 2001 to April 2004 (3 males and 1 female; age: 26 38 years). The duration of heroin abuse was 1.5 10 years (mean: 3.7 years). The course of disease was 5 days in 1 patient, 15 days in 1 and 2 months in 2. In the control group, the brain was collected from 5 dead patients without cerebral lesions in the same period, including 3 cases of sudden cardiac death and 2 cases of pulmonary embolism. There were 4 males and 1 female (age: 51 78 years). Informed consent was obtained from the family members of the deceased. This study was approved by the Ethics Committee of our Hospital.

\section{Instruments}

JEOL JEM-1200EX transmission electron micro- scope (Japan Electronics Company), light microscope (OLYMPUS, Japan) and Anymicro DSS TM/YT-5M Digital shoot system were used in the present study.

\section{Main reagents}

$4 \%$ paraformaldehyde (PFA), TUNEL kit (Nanjing Kagene Biotech Co., Ltd), rabbit anti-human MBP polyclonal antibody (ZA-0186, Zhongshan Golden Bridge), mouse anti-human CD34 monoclonal antibody (Fuzhou Maixin Biotech Co., Ltd) and common secondary antibodies (PV-8000, Zhongshan Golden Bridge) were used in the present study.

\section{Sampling and processing}

The frontal lobe, cerebellum and corpus callosum were collected from the brain and pretreated with $0.5 \mathrm{mmol} / \mathrm{L}$ PBS (pH: 7.3-7.4), $5 \%$ sucrose in PBS at $4^{\circ} \mathrm{C}$ for $2 \mathrm{~h}$ or overnight, $10 \%$ sucrose in PBS at $4^{\circ} \mathrm{C}$ for $1-2$ days and $20 \%$ sucrose in PBS at $4^{\circ} \mathrm{C}$ for 2 days. The brain tissues were cut into sections $(6 \mu \mathrm{m})$ and then attached onto slides. Ten sections were attached to the APES acetone pretreated slides.

\section{Detection of apoptosis by TUNEL staining}

Sections were routinely deparaffinized and hydrated. After washing in PBS, sections were rinsed in $3 \% \mathrm{H}_{2} \mathrm{O}_{2}$ in methanol for $10 \mathrm{~min}$ to inactivate the endogenous peroxidase. Then, these sections were treated with $50 \mu \mathrm{l}$ of $\mathrm{TdT}$ reaction solution (containing $45 \mu \mathrm{l}$ of equilibration buffer, $1 \mu \mathrm{l}$ of biotin-11-dUTP and $4 \mu \mathrm{l}$ of $\mathrm{TdT}$ enzyme) at $37^{\circ} \mathrm{C}$ in dark for $60 \mathrm{~min}$ and with $50 \mu \mathrm{l}$ of streptavidin-HRP working solution (containing $0.5 \mu \mathrm{l}$ of streptavidin-HRP and $99.5 \mu \mathrm{l}$ of PBS) at $37^{\circ} \mathrm{C}$ in dark for $30 \mathrm{~min}$. Visualization was done with $50 \mu \mathrm{l}$ of DAB working solution at room temperature for $10 \mathrm{~min}$. Positive control was prepared according to the manufacturer's instructions to confirm the reliability of testing, and TdT reaction solution was replaced with PBS in the negative control. Under light microscope, 5 fields were randomly selected from each section at a high magnification $(\times 40)$ and the number of TUNEL positive cells in the cortical gray matter, cerebellar white matter and corpus callosum was counted and averaged for statistical analysis.

\section{Immunohistochemistry}

Sections were routinely deparaffinized and hydrated. These sections were blocked in $3 \% \mathrm{H}_{2} \mathrm{O}_{2}$ in $70 \%$ methanol for $10 \mathrm{~min}$ to inactivate endogenous peroxidase. Antigen retrieval was done in $0.1 \mathrm{M}$ sodium citrate buffer ( $\mathrm{pH} \mathrm{6.0)}$ ) at $95^{\circ} \mathrm{C}$ for $13 \mathrm{~min}$. Then, sections were blocked in goat serum working solution (1\% bovine serum albumin [BSA] in PBS, 1:500) at room temperature for $30 \mathrm{~min}$. Then, these sections 
were then treated with rabbit anti-human MBP antibody (1:100) or rabbit anti-human CD34 antibody (1:100) in PBS containing $0.05 \%$ TritonX-100 and $1 \%$ BSA in a humidified environment at $4^{\circ} \mathrm{C}$ overnight and then with secondary antibody. Visualization was done with $\mathrm{DAB}$. In the negative controls, primary antibody or secondary antibody was not added. The positive control was prepared according to the manufacturer's instructions $[7,8]$.

\section{Masson's trichrome staining}

Sections were deparaffinized and processed for HE staining. Then, these sections were fixed in Bouin solution for 12-24 h, Weigert's iron hematoxylin solution for 10-20 min, 1\% hydrochloric acid in ethanol, ponceau acid fuchsin solution for 3-5 min, 1\% phosphomolybdic acid - phosphotungstic acid solution for 5-10 min and aniline blue for 3-5 min. After washing in distilled water, sections were rinsed with $0.5 \%$ glacial acetic acid to remove aniline blue and then treated with 95\% ethanol, absolute ethanol and xylene (twice) followed by mounting with nutral gum.

\section{Detection of demyelination in white matter}

Following immunohistochemistry for MBP, Anymicro image analysis system was employed to capture the representative photographs under a light microscope.

\section{Detection of cerebral microvessels and vascu- lar endothelial cells}

Under a light microscope, image analysis system was employed to examine the sections following Masson's trichrome staining and immunohistochemistry for CD34, and the structure and morphology of cerebral microvessels and endothelial cells were evaluated.

\section{Counting blood vessels}

The single cells or cell groups positive for CD34 were counted regardless of lumen. The positive cells had clear borderline with surrounding blood vessels and tissues. At low magnification $(\times 10)$, the cortical gray matter, cerebellar white matter and corpus callosum were localized, and 5 fields were randomly selected from each section at high magnification $(\times 40)$ followed by counting of blood vessels and averaging.

\section{Statistical analysis}

SPSS version 13.0 for windows was employed for statistical analysis, and data were expressed as mean \pm standard deviation $(\bar{x} \pm \mathrm{s})$. F test was done to compare the number of TUNEL positive cells and that of CD34 positive cells in different cerebral regions in HSLE group and control group. The number of positive cells in the same cerebral region was compared with independent $t$ test between two groups. A value of $\mathrm{P}<0.05$ was considered statistically significant.

\section{Results}

\section{Macroscopy of brains in both groups}

In the control group, the brain was structurally intact. After washing in PBS, the borderline between the gray matter and white matter was clear, and the brain had favorable elasticity. The freshness could be classified as grade $B$.

\section{Immunohistochemistry for MBP}

In the HSLE patients, the myelin sheaths in the subcortical white matter had lighter staining as compared to the control group, the staining was uneven, vacuole-like unstained region was noted, and the number of vacuoles increased with the increase in depth in the brain. In the corpus callosum, fracture or collapse of fibers was found, and vacuoles were also observed. The cerebellar white matter was loose, and a large amount of vacuole-like unstained regions were identified. These findings suggest that there is extensive and diffused vacuole-like demyelination in the cerebral white matter of HSLE patients (Figure 1).
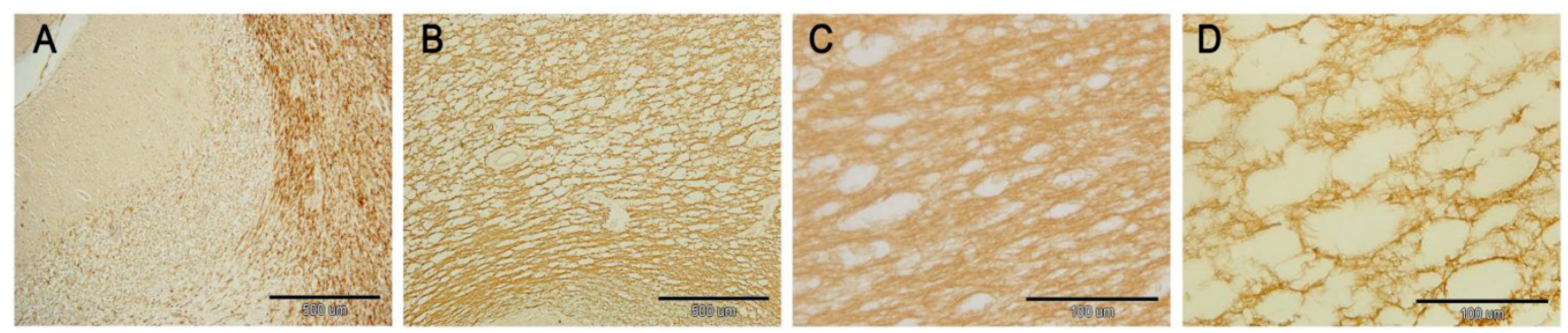

Figure I. Immunohistochemistry for MBP: A: Cerebellum in the control group ( $\times 10)$; B: Cerebellum in the HSLE group $(\times 10)$; C: Myelin sheath was dense in the cerebellar white matter and had clear texture in the control group ( $\times 60)$; D: Myelin sheath was loose, fractured and had disordered structure in the HSLE group $(\times 60)$. 


\section{TUNEL staining}

In the HSLE group, TUNEL staining showed brown cells (apoptotic cells) in the gray matter of frontal lobe, white matter of cerebellum and corpus callosum, and the staining was uneven. Morphologically, chromatin condensation and karyorrhexis occurred, and crescent condensed chromatin was found surrounding the nucleus. The number of TUNEL positive cells in the HSLE group was markedly higher than that in the control group $(F=389.451, P<0.001)$. The number of TUNEL positive cells in the specific region was significantly different between two groups $(F=80.062, P<0.001)$ except in the cerebellum and corpus callosum $(P=0.151)$. However, significant difference was noted between gray matter of frontal lobe and cerebellum / corpus callosum $(P<0.001)$ (Figure 2 and Table 1).

\section{Control}
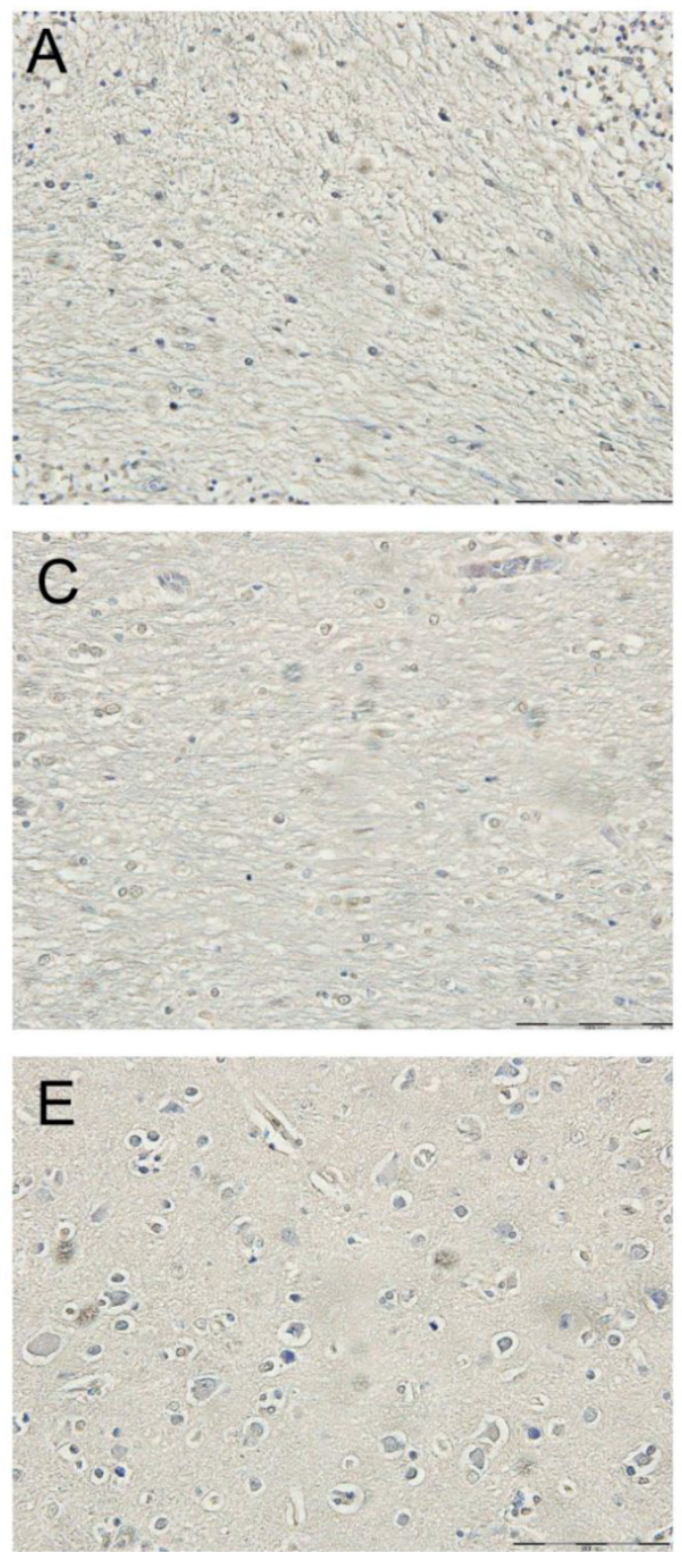

HSLE
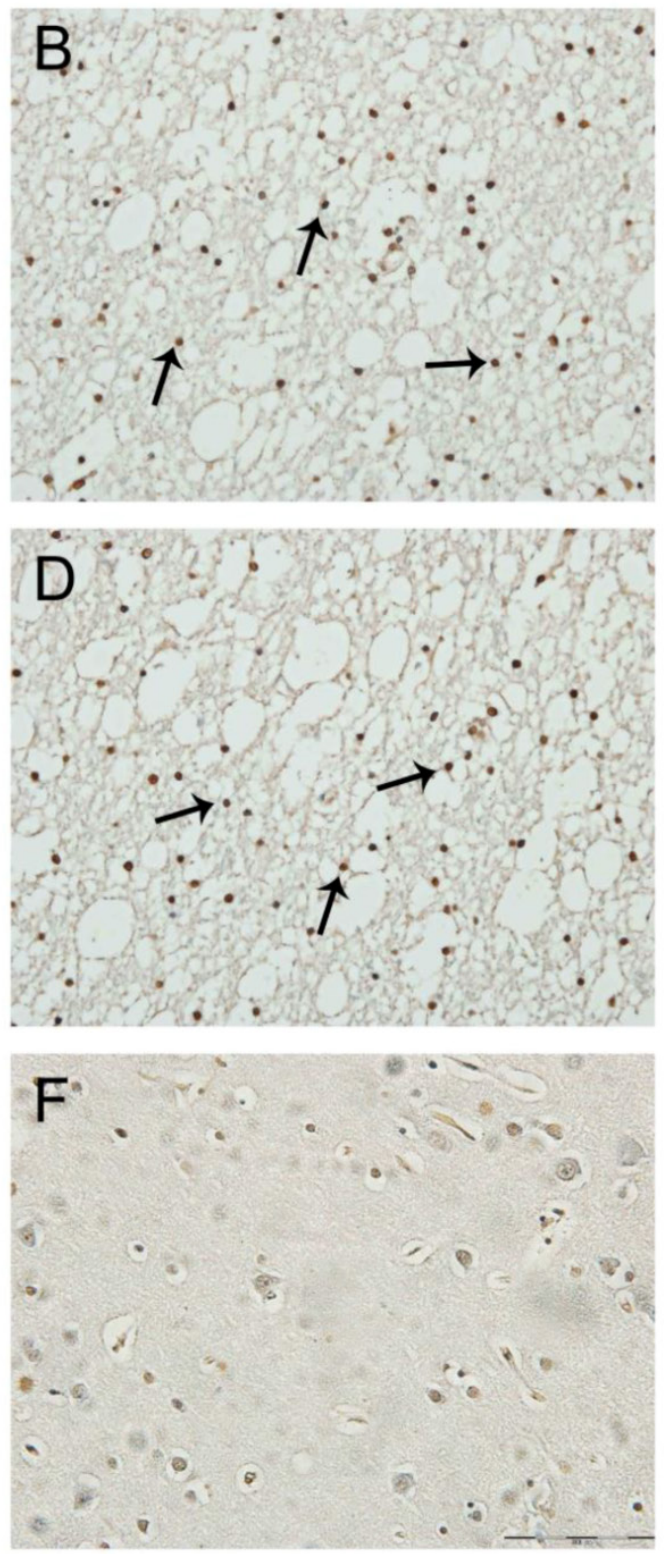

Figure 2. TUNEL staining: $A$ : white matter of cerebellum of control group $(\times 40)$ : no TUNEL positive cells were noted; $B$ : white matter of cerebellum of HSLE group ( $\times 40)$ : a lot of TUNEL positive cells were noted(arrows); C: corpus callosum of control group ( $\times 40)$ : no TUNEL positive cells were noted; D: corpus callosum of HSLE group $(\times 40)$ : a lot of TUNEL positive cells were noted(arrows); E: gray matter of control group $(\times 60)$ : no TUNEL positive cells were noted; F: gray matter of HSLE group $(\times 60)$ : no TUNEL positive cells were noted. 
Table I. TUNEL positive cells and CD34 positive cells in both groups.

\begin{tabular}{lllll}
\hline Group & $\mathrm{n}$ & Frontal lobe & Cerebellum & $\begin{array}{l}\text { Corpus cal- } \\
\text { losum }\end{array}$ \\
\hline $\begin{array}{l}\text { Control } \\
\text { group }\end{array}$ & 5 & & & \\
TUNEL & & $1.92 \pm 0.482$ & $2.32 \pm 0.438$ & $2.12 \pm 0.415$ \\
CD34 & & $12.08 \pm 1.171$ & $10.56 \pm 1.315$ & $10.24 \pm 1.244$ \\
HSLE group & 4 & & & \\
TUNEL & & $4.10 \pm 0.416 \mathrm{a}$ & $29.30 \pm 4.574 \mathrm{a}$ & $25.9 \pm 3.928 \mathrm{a}$ \\
CD34 & & $0.30 \pm 0.258 \mathrm{a}$ & $0.25 \pm 0.252 \mathrm{a}$ & $0.25 \pm 0.191 \mathrm{a}$ \\
\hline
\end{tabular}

Note: a $P<0.01$ vs control group.

\section{Masson's trichrome staining}

Masson's trichrome staining showed extensive vacuolar degeneration was noted in the white matter of HSLE group, and slight vacuoles were found sur- rounding the capillaries in the gray matter of HSLE group. The vacuoles were found mainly around the microvessels and the arterioles while vacuoles around the blood vessels were not obvious in the brain parenchyma of control group

\section{Immunohistochemistry for CD34}

In the HSLE group, no CD34 positive cells were found in the gray matter of frontal lobe, white matter of cerebellum and corpus callosum, and CD34 positive cells were also not identified around the blood vessels. In the control group, a lot of CD34 positive cells were found in the vascular wall of gray matter of frontal lobe, white matter of cerebellum and corpus callosum. There was significant difference in the number of CD34 positive cells between HSLE group and control group $(F=838.500, P<0.001)$, but no significant difference was noted among different regions $(F=2.501, P=0.106)$ (Figure 4 and Table 1$)$.
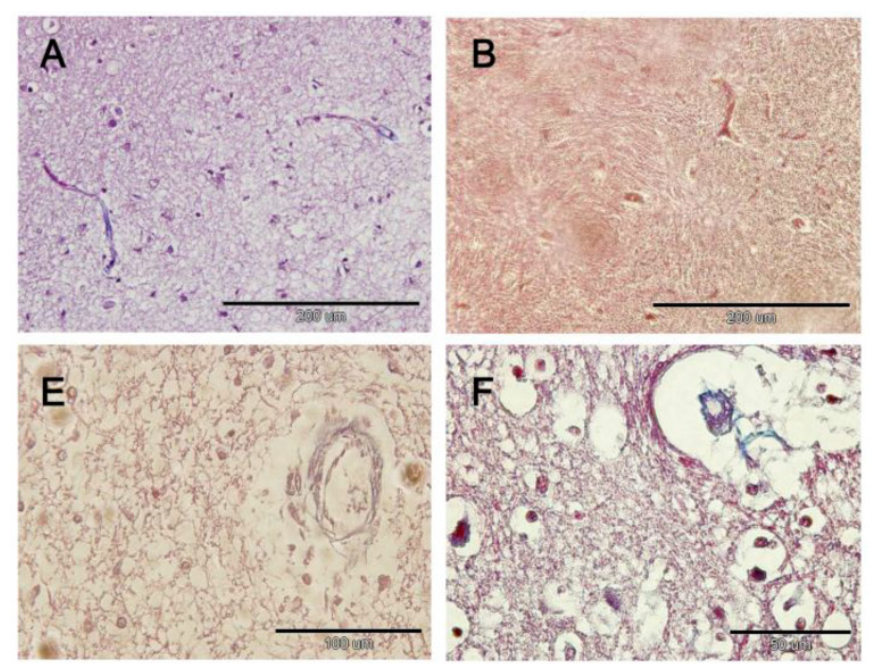
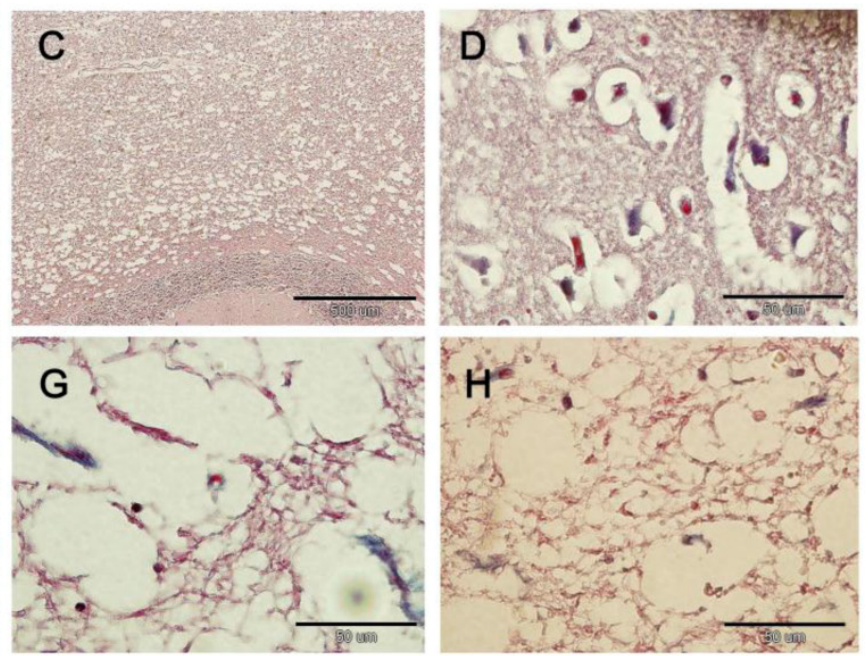

Figure 3. Masson's trichrome staining. A: Gray matter of control group ( $\times 40)$ : the vacuoles in the brain parenchyma and around the blood vessels were not evident, the vascular wall was smooth and intact, had no depression but had even thickness; B: White matter of control group ( $\times 40)$ : brain parenchyma was dense, and vacuoles around the blood vessels were not obvious; C: White matter of cerebellum of HSLE group $(\times 10)$ : extensive vacuolar degeneration was noted; D: Gray matter of frontal lobe of HSLE group $(\times 100)$ : vacuoles were found to surround the capillaries; E: Gray matter of frontal lobe of HSLE group ( $\times 60)$ : vacuoles were not evident around the veins in the gray matter; F: big vacuoles were found around the arterioles of HSLE group $(\times 100)$ and the parenchyma was pushed outward; G and $\mathrm{H}$ : white matter of HSLE group $(\times 100)$ : vacuoles were found around the microvessels (Figure 3 ). 

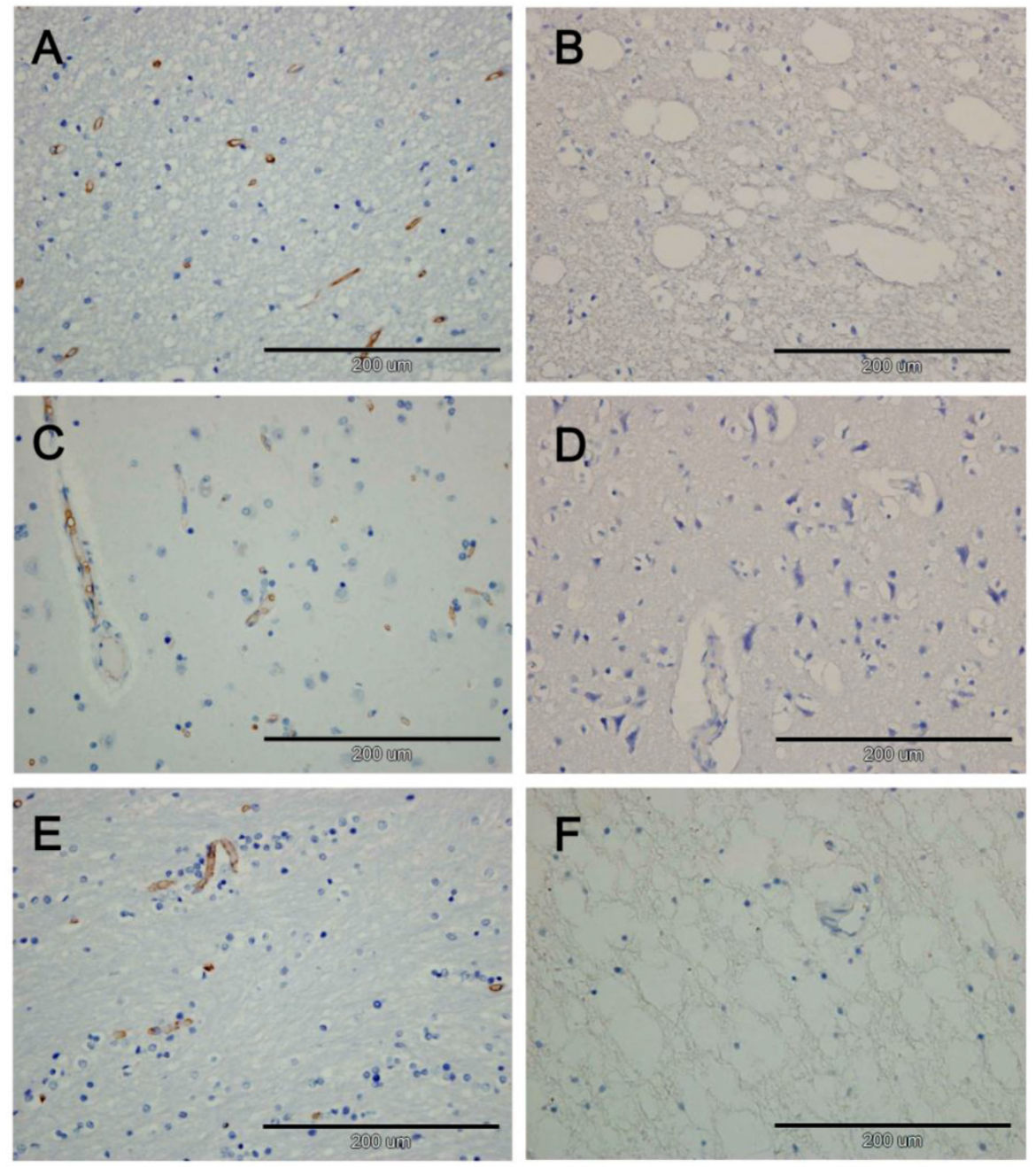

Figure 4. A: white matter of control group, CD34 positive cells were found; $B$ : white matter of cerebellum of HSLE group ( $\times 40)$, CD34 positive cells were not obvious; C: Gray matter of frontal lobe of control group, CD34 positive cells were found along the blood vessels; D: Gray matter of frontal lobe of HSLE group $(\times 40)$ : vacuoles were found around the blood vessels but CD34 positive cells were not noted; E: the corpus callosum of control group was dense, and CD34 positive cells were found in the blood vessels; F: the corpus callosum of HSLE group ( $\times 40)$ : a lot of vacuoles were found, but CD3 positive cells were absent.

\section{Discussion}

HSLE refers to an organic central nervous system disease characterized by spongy vacuolation in cerebral white matter pathologically and by cerebellar ataxia clinically following nasal inhalation of heroin powder [9]. In the present study, immunohistochemistry for MBP was performed to detect the myelin sheath, and results showed the myelin sheaths in the HSLE group were lighter than those in the control group. The vacuoles in the white matter of granular layer were larger, usually oval and distributed along the nerve fibers. These findings suggest the mature myelin sheaths develop lamellar cracking. The vacuoles in the deep white matter were small and dense, which suggests the neuraxon in the deep white matter is slim and the myelin sheath is thin.
These findings indicate that the HSLE is pathologically characterized by the injury of myelin sheaths in the white matter. Oligodendrocytes are the glial cells forming myelin sheath in the CNS. These cells capsulate the axons forming myelin sheaths, and MBP is a major component of myelin sheath. When the integrity of myelin sheath is interrupted pathologically, the MBP expression is reduced [10]. The damage to oligodendrocytes may directly affect the formation of myelin sheaths, and is a major cause of demyelination [4]. These suggest that the spongy vacuolation in the white matter of HSLE patients is essentially the demyelination in the white matter, which may be attributed to the damage to oligodendrocytes.

The electron microscopy of the brain of patients died of stroke has shown the swelling of endothelial cells which protrude into the vascular lumen, and the 
exudation of proteins into the loose brain parenchyma [11]. These findings demonstrate that the progressive damage to the white matter is not caused by the axonal degeneration following stroke, but by the pathological changes in small blood vessels, especially the microvessels. Thus, the vascular leukoencephalopathy may be an arteriolar disease due to the damage to arterioles [11]. The cerebral ischemia-induced apoptosis is initiated by the apoptosis related signals. With the aid of specific signal transduction, apoptosis related genes are activated and signals are transduced into nuclear endonuclease executing death [12]. TUNEL staining aims to detect the 3'-OH of fractured DNA and the apoptotic cells can be specifically located. Under a microscope, the apoptotic cells are observed and counted. In the normal or proliferative cells, there is no fractured DNA, and thus these are not stained in the TUNEL staining. In the present study, results showed there were a few TUNEL positive cells in the gray matter of the cerebrum of HSLE patients, but numerous TUNEL positive cells were found in the white matter, mainly in the subcortical white matter, corpus callosum and white matter of the cerebellum. There was significant difference in the number of TUNEL positive cells between two groups. In addition, these findings were consistent with results in the morphological detection of demyelination. These suggest that the demyelination in the white matter of HSLE patients is related to the apoptosis of oligodendrocytes. Following ischemia/hypoxia, the metabolism in neuronal cells is disordered, and oligodendrocytes are more sensitive to the ischemia/hypoxia than other neuronal cells. Thus, the oligodendrocytes become apoptotic, and fracture and loss of myelin sheath and presence of vacuoles occur subsequently.

There is evidence showing that ischemia/hypoxia may cause the influx of calcium into the axon with myelin sheath, leading to the activation of calcium dependent proteolytic enzymes. These enzymes can degrade the MBP resulting in its reduction [13]. Damage to the cerebral microvessels may cause reduction in the blood flow in the watershed region and increase the hydrolysis of MBP leading to the damage to the myelin sheath in the white matter [10]. This might be another cause of demyelination in the white matter following ischemia/hypoxia.

In HSLE patients, extensive vacuolar degeneration was noted in the cerebral white matter, cerebellar white matter and corpus callosum, which was closely related to the changes in blood vessels. Especially, there were vacuole-like non-staining regions around the arterioles and capillaries, and the surrounding area of vacuoles was irregularly round. In the cortex, there were also vacuoles surrounding the small vessels and capillaries. Immunohistochemistry for macromolecules (such as eNOS and CD34) secreted by vascular endothelial cells has been used as a common technique to investigate the microvessels. In HSLE patients, the CD34 positive cells were not evident in the gray matter of cerebrum, white matter of cerebellum and corpus callosum, which was markedly different from that in the control group. The reduction or even loss of CD34 positive cells suggests the damage to the vascular endothelial cells in the HSLE patients. It is speculated that the cerebrovascular impairment may lead to the microcirculation dysfunction and cerebral ischemia/hypoxia. However, the oligodendrocytes in the white matter are more sensitive to ischemia/hypoxia than other neuronal cells [14], and thus severe vacuolar degeneration is present in the white matter of patients with HSLE at an early stage.

\section{Conclusion}

Apoptosis of oligodendrocytes may be one of pathogenic mechanisms of HSLE. HSLE patients present with changes in the cerebral blood vessels, which might be a major cause of HSLE. Oligodendrocytes are more sensitive to chronic ischemia/hypoxia than other cells, and poor blood supply to the brain may induce the apoptosis of oligodendrocytes resulting in demyelination and spongy vacuolation in the white matter. The interrelation between the apoptosis of oligodendrocytes and the microvascular damage needs to be further studied.

\section{Acknowledgement}

The study was supported by Natural Science Foundation of Guangdong Province (S2011040003054); Medical Research Fund of Guangdong Province (A2011366).

\section{Competing Interests}

The authors have declared that no competing interest exists.

\section{References}

[1] Zhou L, Lu BX, Yin J. Clinical and neuroimaging features of heroin spongiform leukoencephalopathy. Nan Fang Yi Ke Da Xue Xue Bao, 2009; 29(12): 2464-7.

[2] J Yin, SY Pan, L Zhou, et al. Pathological analysis of heroin spongiform leukoencephalopathy. Nan Fang Yi Ke Da Xue Xue Bao, 2007; 27(6): 881-3.

[3] Q wang, BX Lu. Single photon emission computerized tomography of spongiform leukoencephalopathy heroin addicts: analysis of 10 cases. Journal of First Military Medical University, 2002; 22(7):659-660.

[4] A Romero, E Sola, V Lopez, et al. Treatment with thymoglobulin as the cause of acute demyelinating polyneuropathy in a renal transplant patient. Nefrologia, 2009; 29(4): 369-70.

[5] QZ Wu, Q Yang, HS Cate, et al. MRI identification of the rostral-caudal pattern of pathology within the corpus callosum in the cuprizone mouse model. J Magn Reson Imaging. 2008; 27(3): 446-53. 
[6] Chui DH, Dobo E, Makifuchi T, et al. Apoptotic neurons in Alzheimer's disease frequently show intracellular $A \beta 42$ labeling. J Alzheim Dis. 2001;3:231-239.

[7] Jiangyang L, Qian L, Xiaohong W, Yi Y, Ling L, Yi L, Jia Y, Marotta F, Dehua C. Changes of spleen dendritic cells in the terminal stage of multiple organ dysfunction syndrome. Acta Biomed. 2011; 82(2): 146-53.

[8] Chui DH, Tabira T, Izumi S, Koya G, Ogata J. Decreased $\beta$-Amylioid and Increased Abnormal Tau Deposition in the Brain of Aged Patients with Leprosy. Am J Pathol, 1994; 145(4):771-775.

[9] Zhou L, Lu BX, Yin J. Association of cytochrome P4502D6 gene polymorphism with the susceptibility of heroin spongiform leucoencephalopathy. Nan Fang Yi Ke Da Xue Xue Bao, 2010; 30(3): 572-583.

[10] Pernet V, Joly S, Christ F, Dimou L, Schwab ME. Nogo-A and Myelin-Associated Glycoprotein Differently Regulate Oligodendrocyte Maturation and Myelin Formation. J. Neurosci. 2008; 28: 7435-7444.

[11] Dziewulska D, Rafalowska J, Podlecka A, et al. Remote morphological changes in the white matter after ischaemic stroke. Folia Neuropathol, 2004;42(2): 75-80.

[12] Moraru M, Egger B, Bao DB, et al. Analysis of cell identity, morphology, apoptosis and mitotic activity in a primary neural cell culture system in Drosophila. Neural Dev. 2012; 7(1): 14

[13] Miki K, Ishibashi S, Sun L, et al. Intensity of chronic cerebral hypoperfusion determines white/gray matter injury and cognitive/motor dysfunction in mice. J Neurosci Res. 2009; 87(5): 1270-81.

[14] PetitoCK, Olarte JP, RobertsB, et al. Selective glial vulnerability following transient global ischemia in rat brain. Neuropathol Exp Neurol 1998; 57(3): 231-238. 